\title{
Isolated lymphoma of the optic nerve, chiasm and tract: A case report
}

\author{
YUE-LI ZHU ${ }^{1,2}$, XIU-FENG SONG ${ }^{3}$ and BIN WANG ${ }^{1,4}$ \\ ${ }^{1}$ Shandong Medical Imaging Research Institution, Shandong University, Jinan, Shandong 250021; \\ ${ }^{2}$ Department of Radiology, Qingdao Municipal Hospital, Qingdao, Shandong 266071; \\ ${ }^{3}$ Department of Radiology, Qingdao University Affiliated Hospital, Qingdao, Shandong 266003; \\ ${ }^{4}$ Medical Imaging Research Institute, Binzhou Medical University, Yantai, Shandong 264003, P.R. China
}

Received October 16, 2014; Accepted July 17, 2015

DOI: $10.3892 / \mathrm{ol} .2015 .3675$

\begin{abstract}
The current study reports the case of a 68-year-old, previously healthy female who presented with progressive visual impairment leading to blindness bilaterally. Brain imaging features were suggestive of malignant glioma of the anterior visual pathway. Postoperative examination indicated a diagnosis of diffuse malignant lymphoma type B. As no evidence of extracranial lymphoma was observed, the final diagnosis was primary central nervous system lymphoma (PCNSL). Following treatment with surgery and radiotherapy, the patient's symptoms went into remission. At a follow-up examination 12 months after diagnosis, the patient demonstrated no evidence of recurrence. To the best of our knowledge, PCNSL isolated to the optic chiasm has been reported only three times in immunocompetent patients. Therefore, the present case of the lymphoma involving the optic nerve, optic chiasm and optic tract in an immunocompetent patient is unusual. The present case emphasizes the importance of considering the diagnosis of lymphoma in this setting.
\end{abstract}

\section{Introduction}

Primary central nervous system lymphoma (PCNSL) is an uncommon intracranial lesion that accounts for $<0.7 \%$ of all malignant lymphomas and $<1 \%$ of intracranial tumors (1). This disease occurs more frequently in patients who are immunocompromised, including those with diseases such as acquired immune deficiency syndrome (AIDS), and rarely occurs in immunocompetent patients (1). In the immunocompetent population, PCNSLs typically appear in older patients in their fifties and sixties. However, the incidence of PCNSL

Correspondence to: Dr Xiu-Feng Song, Department of Radiology, Qingdao University Affiliated Hospital, 16 Jiangsu Road, Qingdao, Shandong 266003, P.R. China

E-mail: song-sxf@163.com

Key words: magnetic resonance imaging, lymphoma, optic nerve, computed tomography in the immunocompetent population has been reported to have increased $>10$-fold from 2.5 cases to 30 cases per 10 million population between 1973 and $1992(2,3)$. The cause for the increase in incidence of this disease in the immunocompetent population is unknown. Surgical resection is generally ineffective due to the depth of the tumor. Furthermore, treatment with radiotherapy and corticosteroids often only produce a partial response, and tumors recur in $>90 \%$ of patients (4). Median survival is $10-18$ months in immunocompetent patients, and less in those with AIDS (5). There have been few reports of PCNSL isolated to the optic nerve without infiltration of the brain, retina or vitreous, and in the absence of extracranial signs of non-Hodgkin lymphoma (NHL) $(1,6-8)$.

The current study reports a case of isolated lymphoma of the optic nerve, optic chiasm and optic tract in an immunocompetent patient, in whom the diagnosis was determined postoperatively.

\section{Case report}

A 68-year-old female was referred to the neuro-ophthalmology clinic of Qingdao Municipal Hospital (Qingdao, China) with bilateral progressive painless loss of vision over a period of one year leading to blindness in June 2014. The patient's medical history included open cavity drum-type tympanitis surgery to the left ear for middle ear cholesteatoma $>30$ years previously and a resulting fistula with occasional discharge. No history of high blood pressure, coronary heart disease or diabetes mellitus was noted. The patient did not report experiencing any headaches, fever, nausea, vomiting or weight loss.

The patient developed progressive visual loss in the right eye for one year without ophthalmalgia, redness or swelling, and this did not improve following intermittent treatment with esculin and digitalis glycosides eye drops (one drop, three time a day) for one year. Blindness had developed in the right eye around 6 months prior to the present admission. Decreased vision was also reported in the left eye, which did not improve with treatment, and the left eye had developed blindness 3 days previously. The patient also reported a sensation of numbness in the legs. Physical examination revealed no light perception in either eye, and direct and indirect light reflexes had disappeared. Hearing loss was noted in the left ear, and limb muscle 

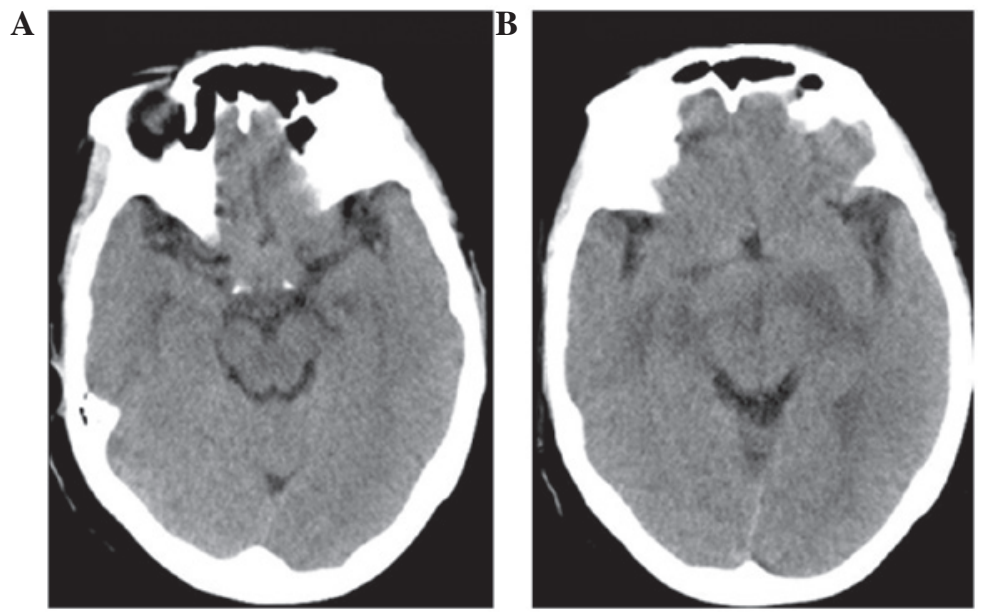

Figure 1. Axial brain plain computed tomography. A suprasellar isodensity mass involving (A) the optic chiasm, both optic nerves and (B) the right optic tract with surrounding edema was observed.
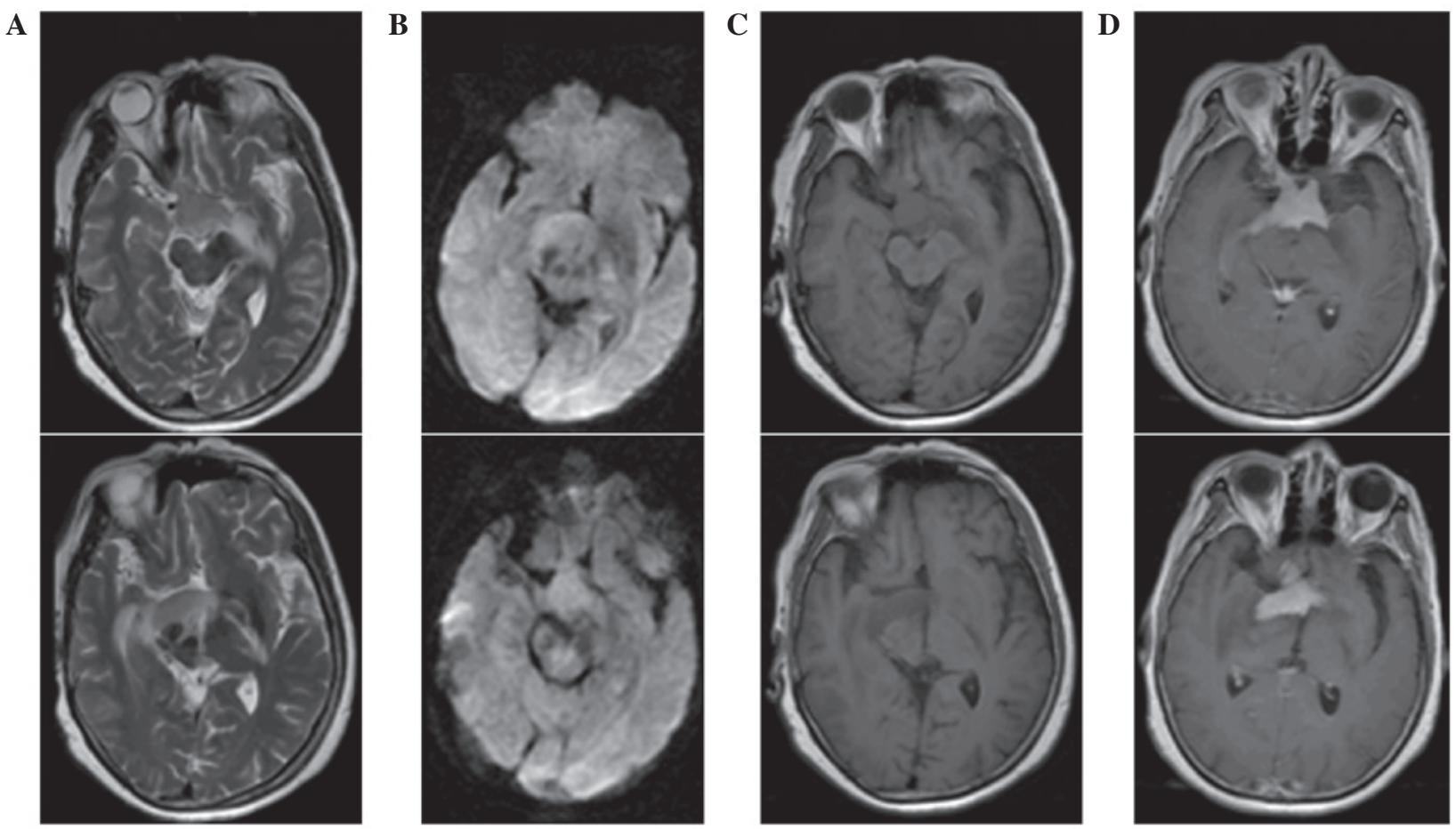

Figure 2. Axial brain magnetic resonance imaging: (A) T2WI; (B) DWI; (C) precontrast T1; (D) postcontrast T1. A suprasellar mass with significant homogenous enhancement involving the optic chiasm and both optic nerves (top row) and right optic tract (bottom row) was observed. The mass exhibited iso-intensity to the cortex on T1WI and T2WI, and slight hyper-intensity on DWI. As on the T2WI, the signal changes (B) were more extensive than the enhancement (D) owing to lesional edema. T2WI, T2-weighted imaging; DWI, diffusion-weighted imaging; T1WI, T1-weighted imaging.

strength was determined to be grade 4 (active movement against resistance and gravity), according to the Manual Muscle Testing grading system (9). A blood test revealed that triiodothyronine (T3) $(1.27 \mathrm{nmol} / 1$; normal range, $1.34-2.73 \mathrm{nmol} / \mathrm{l})$, thyroxine (T4) $(64.33 \mathrm{nmol} / \mathrm{l}$; normal range, $78.38-157.4 \mathrm{nmol} / \mathrm{l})$, free T3 (FT3) $(3.18 \mathrm{nmol} / \mathrm{l}$; normal range, $3.8-6.0 \mathrm{nmol} / \mathrm{l})$ and FT4 (7.09 pmol/1; normal range, 7.85-14.41 pmol/1) levels were reduced, indicating thyroid hypofunction; the results of other laboratory examinations were within the normal limits, and the HIV titer was negative.

Computed tomography (CT) of the head revealed a suprasellar isodensity mass (Fig. 1). In addition, magnetic resonance imaging (MRI) of the head demonstrated a suprasellar mass with significant homogenous enhancement and involvement of the optic chiasm, spreading along the two optic nerves and the right optic tract (Figs. 2 and 3); iso-signal intensity to the cortex was observed on T1- and T2-weighted imaging (WI), and slight hyperintensity on diffusion-weighted imaging (DWI).

Surgery revealed a thickening of the right optic nerve, with a diameter of $1 \mathrm{~cm}$. The optic chiasm also appeared thickened, at $2-3 \mathrm{~cm}$. The tumor was dark red in appearance, with a well-defined boundary. Pathological examination of a resected section of tumor indicated malignancy; complete resection of 
A

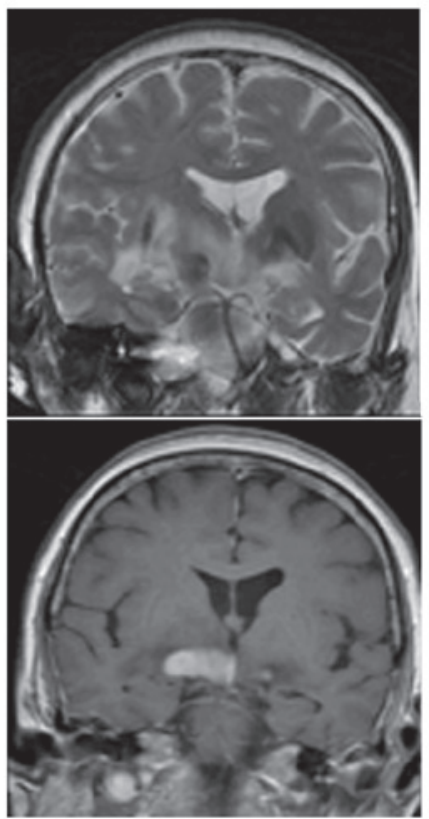

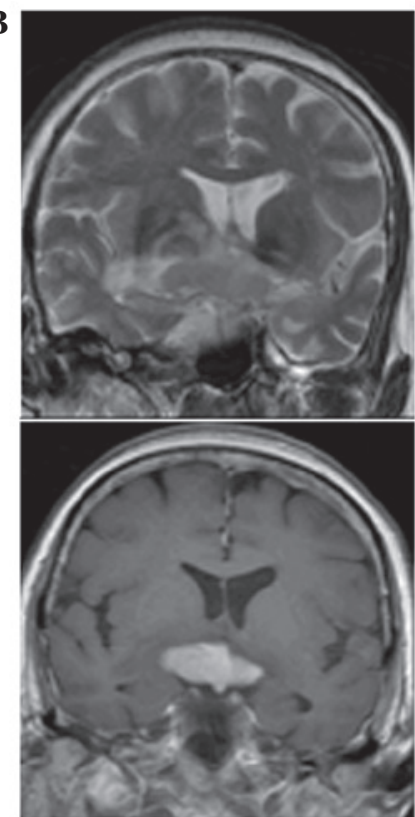
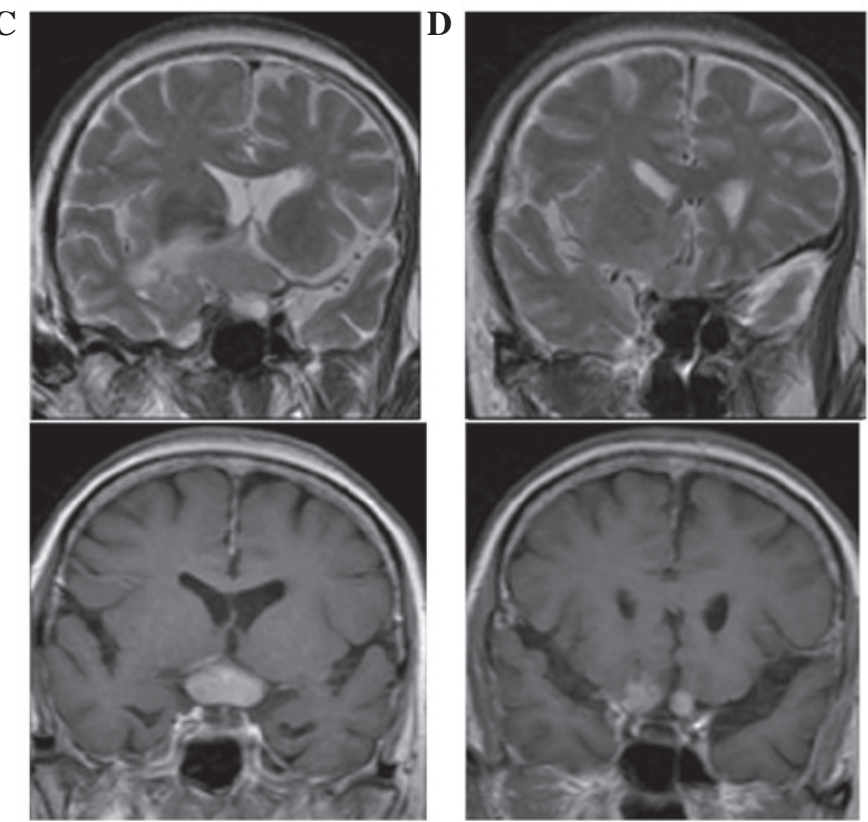

Figure 3. Coronal brain magnetic resonance imaging: Top row, T2-weighted imaging; bottom row, post-contrast T1-weighted imaging. The suprasellar mass showed significant enhancement involving (A) right optic tract, (B and C) optic chiasm, and (D) both optic nerves.
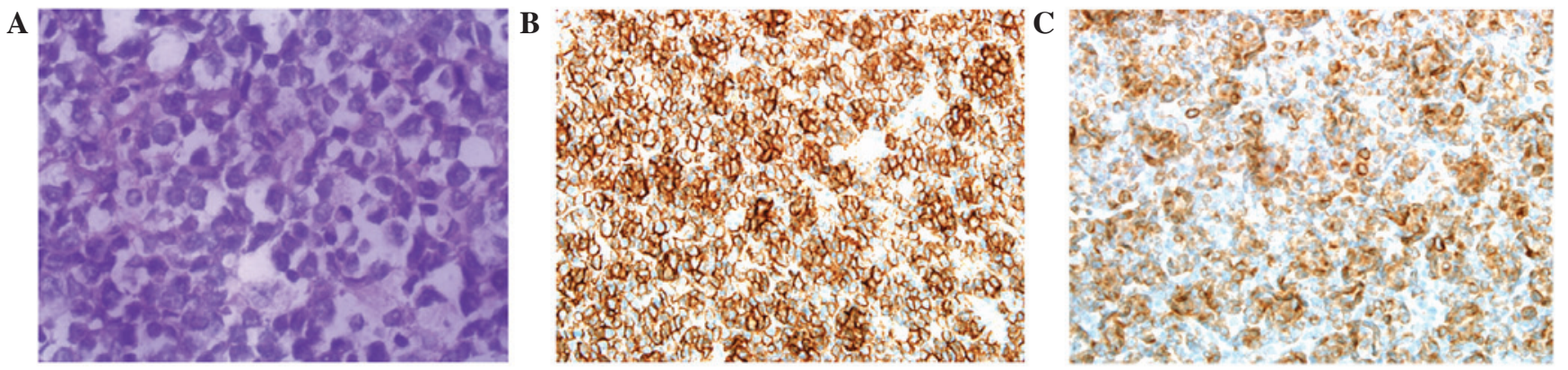

Figure 4. Pathological analysis of the resected tumor. (A) The densely cellular tumor showed frequent mitotic figures and surrounded blood vessels (hematoxylin and eosin staining). (B) Cluster of differentiation (CD) 20(+) (hematoxylin staining). (C) CD79a(+) (hematoxylin staining; original magnification, x400).

the tumor was then performed. Pathology confirmed diffuse malignant lymphoma type B in the anterior visual pathway: Hematoxylin and eosin staining revealed macronuclei, less cytoplasm and thickened chromatin granules, without hemorrhage, necrosis and calcifications. Immunohistochemical staining revealed that the tissue was positive for leukocyte common antigen, B lymphocyte marker CD20 and B-cell antigen receptor Ig $\alpha \mathrm{CD} 79 \mathrm{a}$, and negative for $\mathrm{T}$ cell marker CD3 and glial fibrillary acidic protein (Fig. 4).

Postoperative CT of the neck, chest, abdomen and pelvis revealed no evidence of systemic lymphoma or other medical conditions. Therefore, the final diagnosis of was PCNSL.

The tumor was completely resected prior to the administration of radiotherapy (50 Gy in 25 fractions) for five weeks and eight three-week cycles of the R-CHOP chemotherapy regimen $\left(375 \mathrm{mg} / \mathrm{m}^{2}\right.$ rituximab, day 1 ; $750 \mathrm{mg} / \mathrm{m}^{2}$ cyclophosphamide, day $1 ; 50 \mathrm{mg} / \mathrm{m}^{2}$ doxorubicin, day $1 ; 1.4 \mathrm{mg} / \mathrm{m}^{2}$ vincristine, day $1 ; 40 \mathrm{mg} / \mathrm{m}^{2}$ prednisone, days 1-5). The initial response to radiotherapy was excellent and partial remission was achieved following the completion of eight cycles of the R-CHOP chemotherapy regimen.
The patient was followed-up by telephone interview, every three months for one year. At a follow-up examination 12 months after diagnosis, the patient demonstrated no evidence of recurrence; however, 14 months after diagnosis the patient succumbed to the disease.

This study was conducted in accordance with the declaration of Helsinki and with approval from the Ethics Committee of Qingdao University. Written informed consent was obtained from the patient.

\section{Discussion}

Imaging findings of PCNSL. Anterior visual pathway involvement from lymphoma may be divided into three categories: Primary intraocular lymphoma, PCNSL and secondary metastatic involvement from systemic NHL (1). PCNSL is predominantly of $\mathrm{B}$ cell origin and is associated with invasion and poor prognosis (10). A number of MRI findings may be important in the diagnosis of intracranial PCNSL: i) PCNSL is often observed in the form of focal or multifocal lesions of the cerebral hemispheres with involvement of the cortical 
white-matter junction, the basal ganglia, thalamus and periventricular areas (11); ii) as PCNSL has a high cell density and high nucleus:cytoplasm ratio on pathology, it may be similar to other high cell density tumors, such as meningioma, which exhibit isodense or hyperdense on CT signals and signal intensity close to the cortical gray matter on T1WI and T2WI MRI (12). However, gliomas often exhibit hypodensity on CT and hyperintensity on T2WI MRI; iii) PCNSLs typically exhibit high signal intensity on DWI, as their compact tumor cells, small extracellular spaces and high nucleus:cytoplasm ratios restrict diffusion of water in the tumor (13); iv) PCNSLs exhibit moderate homogeneous enhancement with irregular edges, which may be associated with the tumor growth along the perivascular space and easy aggregation of tumor cells (14); v) multifocal lesions often have regional distribution, and cystic necrosis is rare (15); and vi) tumors are sensitive to radiotherapy, and steroid therapy, practical diagnostic radiotherapy and chemotherapy of suspected cases may aid in determining a clinical diagnosis if the lesions shrink or disappear obviously and rapidly (5).

PCNSL may also involve the intraorbital or intracranial optic nerves, chiasm, radiations or visual cortex. In addition, studies have reported cavernous sinus or superior orbital fissure involvement and direct infiltrative or compressive lesions (e.g., optic nerve, chiasm and tract) (16-22). Lymphoma involving the chiasm typically occurs due to metastatic spread of systemic NHL (23-26). PCNSL isolated to the optic chiasm is extremely rare. Zelefsky et al (7) reported a case of PCNSL in a 72-year-old previously healthy man. Brain MRI revealed abnormal contrast enhancement of the intracranial portion of the right optic nerve and optic chiasm, which extended posteriorly to the right optic tract and toward the left lateral geniculate body, with the MRI signal intensity close to that of the cortex (7). Lee et al (1) reported a case of PCNSL involving the optic chiasm in a patient with AIDS. In the current case, a suprasellar mass with equal density was identified on CT. The imaging features included isodensity on plain $\mathrm{CT}$, isointensity to the cortical gray matter on T1WI and T2WI, and hyperintensity DWI on plain MRI, while homogeneously marked enhancement of the optic chiasm, which extended along both optic nerves and the right optic tract with well-defined boundaries, was observed on enhanced MRI. The imaging features of the mass involving the optic chiasm in the current case were generally consistent with the features of intracranial PCNSL involving other locations as mentioned above, which suggested the possibility of lymphoma.

Differential diagnosis. With regard to differential diagnosis, there are a number of other conditions that may be considered. The optic nerve, optic chiasm and optic tract are collectively known as anterior visual pathway (27). Whilst the optic nerve comprises myelinated nerve fibers, no Schwann membranes are present; therefore, Schwann cell tumors (Schwannomas) seldom occur in the visual pathway (28). Meningiomas of the optic chiasm are also extremely rare, and typically arise from the expansion of optic nerve sheath meningiomas (29).

Astrocytomas are the most common tumors of the optic nerve and chiasm, and may be divided into two groups according to the age of the patient: A pediatric group and an adult group. Pediatric astrocytomas are more common; pilocytic astrocytoma is the most common astrocytoma, and such tumors predominantly occur in children $<10$ years of age. In adults, astrocytomas are less common, however, the degree of malignancy is higher than that in children, and these cases are most frequently anaplastic astrocytoma or glioblastoma (30). An association between optic nerve glioma and neurofibromatosis type I is well established (31). On MRI, an enlarged optic chiasm, or the formation of mass with a clear boundary may be observed. The lesions exhibit hypodensity on CT, whereas equal or high density is observed with PCNSL, and hypointensity on T1WI and hyperintensity on T2WI, with mild or significant enhancement, by contrast to the isosignals to the cortex observed on T1WI and T2WI with significant enhancement in PCNSL. Furthermore, hyposignal is observed on DWI, whereas high-signals are exhibited with PCNSL. The cystic lesions can migrate from the tumor (more common in malignant tumors) (30).

Optic chiasm metastases are rare and predominantly result from intracranial invasion of retinoblastoma (29). This may appear as a mass spreading along the optic nerve to the optic chiasm, with an enlarged optic nerve and chiasm. Distant metastasis to the optic chiasm is very rare unless the patient has a relevant history of malignant tumor. MRI may reveal thickening of the optic chiasm with homogeneous and significant enhancement (32).

Conclusions. Imaging findings of tumors in the optic chiasm are often similar and it may be difficult to differentiate between them (29). Gliomas (primarily astrocytic-origin tumors) are the most common tumor and should therefore be considered first (30). Combination of medical history indicating no evidence of other malignant tumors can help to exclude metastases. Lymphoma in the optic chiasm is extremely rare; however, optic chiasm tumors with marked homogeneous enhancement and signals close to the cortex on T1WI and T2WI, and hyperintensity on DWI in adults, may indicate lymphoma (1-3,6,7). The inflammatory lesion of the optic chiasm shows diffuse thickening and significant enhancement, as it is often associated with inflammation of the surrounding structure, commonly involving the adjacent dura (29).

In the current case involving relatively rapid and progressive visual impairment in both eyes in a previously healthy female and imaging abnormalities, surgery revealed lymphoma. This case demonstrates the importance of considering such a diagnosis in this setting.

\section{References}

1. Lee AG, Tang RA, Roberts D, Schiffman JS and Osborne A: Primary central nervous system lymphoma involving the optic chiasm in AIDS. J Neuroophthalmol 21: 95-98, 2001.

2. Eby NL, Grufferman S, Flannelly CM, Schold SC, Vogel FS and Burger PC: Increasing incidence of primary brain lymphoma in the US. Cancer 62: 2461-2465, 1988.

3. Corn BW, Marcus SM, Topham A, Hauck W and Curran WJ: Will primary central nervous system lymphoma be the most frequent brain tumor diagnosed in the year 2000? Cancer 79: 2409-2413, 1997.

4. Deangelis LM and Hormigo A: Treatment of primary central nervous system lymphoma. Semin Oncol 31: 684-692, 2004.

5. Sierra del Rio M, Rousseau A, Soussain C, Ricard D and Hoang-Xuan K: Primary CNS lymphoma in immunocompetent patients. Oncologist 14: 526-539, 2009. 
6. Behbehani RS, Vacarezza N, Sergott RC, Bilyk JR, Hochberg F and Savino PJ: Isolated optic nerve lymphoma diagnosed by optic nerve biopsy. Am J Ophthalmol 139: 1128-1130, 2005.

7. Zelefsky JR, Revercomb CH, Lantos G and Warren FA: Isolated lymphoma of the anterior visual pathway diagnosed by optic nerve biopsy. J Neuroophthalmol 8: 36-40, 2008.

8. Cantore GP, Raco A, Artico M, Ciappetta P and Delfini R: Primary chiasmatic lymphoma. Clin Neurol Neurosurg 91: 71-74, 1989.

9. Simon RP, Greenberg D and Aminoff MJ: Clinical Neurology. 7th edition. McGraw-Hill Medical, New York, NY, 2009.

10. Chimienti E, Spina M, Vaccher E and Tirelli U: Management of immunocompetent patients with primary central nervous system lymphoma. Clin Lymphoma Myeloma 9: 353-364, 2009.

11. Bühring U, Herrlinger U, Krings T, Thiex R, Weller M and Küker W: MRI features of primary central nervous system lymphomas at presentation. Neurology 57: 393-396, 2001.

12. Küker W, Nägele T, Korfel A, Heckl S, Thiel E, Bamberg M, Weller $\mathrm{M}$ and Herrlinger U: Primary central nervous system lymphomas (PCNSL): Presentation in 100 patients. J Neuro Oncol 72: 169-177, 2005.

13. Guo AC, Cummings TJ, Dash RC and Provenzale JM: Lymphomas and high-grade astrocytomas: Comparison of water diffusibility and histologic characteristics. Radiology 224 177-183, 2002

14. Go JL, Lee SC and Kim PE: Imaging of primary central nervous system lymphoma. Neurosurg Focus 21: E4, 2006.

15. Haldorsen IS, Espeland A and Larsson EM: Central Nervous system lymphoma: Characteristic findings on traditional and advanced imaging. AJNR Am J Neuroradiol 32: 984-992, 2011.

16. Ikeda T, Hara K, Yamanaka T, Umezu H, Takahashi H and Nishizawa M: A case of primary central nervous system malignant lymphoma developing from the optic chiasma and hypothalamus Rinsho Shinkeigaku 46: 475-479, 2006 (In Japanese).

17. Bullock JD, Yanner B, Kelly M and McDonald LW: Non-Hodgkins lymphoma involving the optic nerve. Ann Ophthalmol 11: 1477-1480, 1979

18. Maiuri F: Primary cerebral lymphoma presenting as steroid-responsive chiasmal syndrome. Br J Neurosurg 1: 499-502, 1987

19. Sakai C, Takagi T and Wakatsuki S: Primary meningeal lymphoma presenting solely with blindness: A report of an autopsy case. Int J Hematol 63: 325-329, 1996.

20. Strominger MB, Schatz NJ and Glaser JS: Lymphomatous optic neuropathy. Am J Ophthalmol 116: 774-776, 1993.
21. Gray RS, Abrahams JJ, Hufnagel TJ, Kim JH, Lesser RL and Spencer DD: Ghost-cell tumor of the optic chiasm: Primary CNS lymphoma. J Clin Neuroophthalmol 9: 98-104, 1989.

22. Maiuri F: Visual involvement in primary non-Hodgkin's lymphomas. Clin Neurol Neurosurg 92: 119-124, 1990.

23. Bolanowski M, Kuliszkiewicz-Janus M and Sokolska V: Diffuse malignant lymphoma type B with optic chiasm infiltration, visual disturbances, hypopituitarism, hyperprolactinaemia and diabetes insipidus. Case report and literature review. Endokrynol Pol 57: 642-647, 2006

24. McFadzean RM, McIlwaine GG and McLellan D: Hodgkin's disease at the optic chiasm. J Clin Neuroophthalmol 10: 248-254, 1990.

25. Sumrall A and Herrin V: Recurrent, transformed non-Hodgkin's lymphoma presenting as chiasmal syndrome with hyperprolactinemia and hypopituitarism. J Miss State Med Assoc 51: 35-36, 2010

26. Soldevilla HF, Molina RM and Navarra SV: Breast lymphoma in Sjögren's syndrome complicated by acute monocular blindness. Int J Rheum Dis 13: 164-170, 2010.

27. Song X, Wang G, Zhang T, Feng L, An P and Zhu Y: Functional magnetic resonance imaging evaluation of visual cortex activation in patients with anterior visual pathway lesions. Neural Regen Res 7: 692-696, 2012.

28. Quesnel AM and Mckenna MJ: Current strategies in management of intracanalicular vestibular schwanmoma. Curr Opin Otolaryngol Head Neck Surg 19: 335-340, 2011.

29. Wu R, Wang Z, Xian J, et al: Analysis of the optic chiasmal lesions by MRI. Zhonghua Fang She Xue Za Zhi 37: 445-448, 2003 (In Chinese).

30. Xian J, Wang Z, Yu W, et al: Imaging investigations of optic gliomas. Zhonghua Fang She Xue Za Zhi 38: 677-681, 2004 (In Chinese).

31. Listernick R, Louis DN, Packer RJ and Gutmann DH: Optic pathway gliomas in children with neurofibromatosis type 1 : Consensus statement from the NF1 Optic Glioma Task Force. Ann Neurol 41: 143-149, 1997.

32. Albert A, Lee BC, Saint-Louis L and Deck MD: MRI of optic chiasm and optic pathways. AJNR Am J Neuroradiol 7: 255-258, 1986. 